\title{
Surface engineering of iron oxide nanoparticles for targeted cancer therapy
}

\author{
Forrest M. Kievit and Miqin Zhang ${ }^{\star}$ \\ Department of Materials Science and Engineering, University of Washington, Seattle, WA 98195, \\ USA
}

\section{Conspectus}

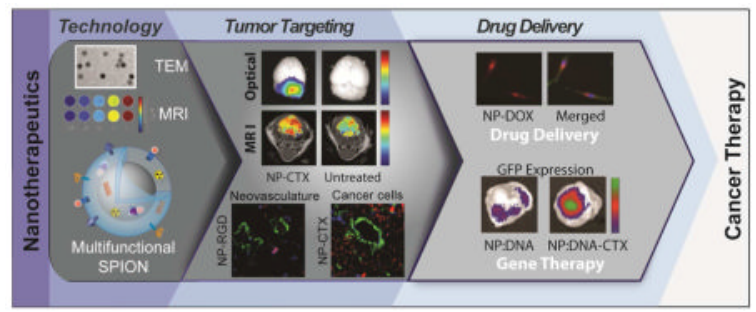

Nanotechnology provides a flexible platform for the development of effective therapeutic nanomaterials that can interact specifically with a target in a biological system and provoke a desired biological response. Of the nanomaterials studied, iron oxide nanoparticles have emerged as one of top candidates for cancer therapy due to their intrinsic superparamagnetism that enables no-invasive magnetic resonance imaging (MRI) and biodegradability favorable for in vivo application. A therapeutic superparamagnetic iron oxide nanoparticle (SPION) typically consists of three primary components: an iron oxide nanoparticle core that serves as both a carrier for therapeutics and contrast agent for MRI, a coating on the iron oxide nanoparticle that promotes favorable interactions between the SPION and biological system, and a therapeutic payload that performs designated function in vivo. Often, a targeting ligand is also included in the design that recognizes the receptors over-expressed on cancer cells. The body is a highly complex system that imposes multiple physiological and cellular barriers to foreign objects. Thus, the success of a therapeutic SPION largely relies on the proper design of the iron oxide core to ensure MRI detectability and more critically, the coating to render the ability to bypass these barriers.

Strategies to bypass the physiological barriers such as liver, kidneys, and spleen, involve tuning the overall size and surface chemistry of the SPION to maximize blood half-life and facilitate the navigation in the body. Strategies to bypass cellular barriers include the use of targeting agents to maximize uptake of the SPION by cancer cells, and employing materials that promote desired intracellular trafficking and enable controlled drug release.

The payload can be genes, proteins, chemotherapy drugs, or a combination of them. Each therapeutic requires a specific coating design to maximize the loading and achieve effective delivery and release. In this Account, we discuss the primary design parameters in developing therapeutic SPIONs with a focus on surface coating design to overcome the barriers imposed by the body's defense system and provide examples of how these design parameters have been implemented to produce therapeutic SPIONs for specific therapeutic applications.

Although there are still challenges to be addressed, SPIONs show great promise in successful diagnosis and treatment of the most devastating cancers. Once critical design parameters have

*To whom correspondence should be addressed. Phone: (206) 616-9356, Fax: (206) 543-3100, mzhang@u.washington.edu. 
been optimized, these nanoparticles, combined with imaging modalities, can serve as a truly multifunctional theranostic agent that not only performs a therapeutic function, but provides instant treatment feedback for the physician to adjust the treatment plan.

\section{Introduction}

Cancers are traditionally treated with surgery, radiation, and chemotherapy. Each of these approaches bears the risk of killing normal cells or fatally damaging healthy tissue. With the emergence and rapid growth of nanotechnology, nanomaterials are providing new opportunities that can assist in the delivery of small-molecule drugs and biological agents to improve the therapeutic index by driving the therapeutic to the target site and limiting its exposure to healthy tissue. Among many nanomaterials studied for cancer diagnosis and therapy, superparamagnetic iron oxide nanoparticles (SPIONs) have emerged as one of the most appealing candidates. With superparamagnetism, SPIONs can be used as a contrast agent in magnetic resonance imaging (MRI) for disease diagnosis and treatment monitoring. Iron oxide is biodegradable and the iron from degraded SPIONs enters the body's natural iron stores such as hemoglobin in red blood cells. ${ }^{1}$ In fact, several SPION formulations have been approved for clinical use including Ferridex I.V. ${ }^{\circledR}$ for liver and spleen imaging, Ferumoxytol ${ }^{\circledR}$ for iron replacement therapy, and Combidex ${ }^{\circledR}$ for imaging lymph node metastases. The small size of SPIONs ensures the overall small size of the resultant therapeutic SPION, which facilitates their navigation in tissues, endocytosis, and intracellular trafficking in cancer cells.

Figure 1 illustrates the architecture of a typical multi-functional SPION system. A SPION core is coated with a biocompatible coating that allows integration of multiple functionalities into a single particle. The large surface-to-volume ratio allows high-capacity drug loading, and attachment of targeting ligands for tumor-specific drug delivery and membrane permeating molecules for intracellular trafficking. By presenting a targetsensitive biomolecule at the SPION surface, it is possible to create a library of novel, personalized therapeutics that can be used to treat a variety of diseases. One of the most exciting areas of the development is in combination with gene therapies. Use of many emerging biological drugs, such as siRNA and plasmid DNA, has been hindered by the inability to pass the therapeutics to the cellular compartment of target cells without first being damaged by nucleases. Nanoparticle-based carriers can be used to transport these genes and release them at the sites where their therapeutic effects are intended. In addition, the superparamagnetic core itself can be used for therapy through hyperthermia.

\section{Design considerations}

At the core of nanotherapeutic agent design is the identification of materials and techniques that can be used to create the end-product that meets the rigorous requirements for in vivo trafficking, monitoring and therapeutic function. The component materials for a nanotherapeutic agent are normally chosen for biocompatibility, including the degradation products, while being able to perform their designated functions. This can be a complex process because these materials are to interact with biological systems, and our current understanding in material-biosystem interaction is limited. The techniques and approaches used to assemble these materials together must maintain the integrity of the end-product during its navigation through the body until it reaches target tissue or cells. The interplay of these components can substantially complicate the design implementation. Here we discuss the principal design considerations in development of therapeutic SPIONs. 


\subsection{Superparamagnetic core}

The miniaturization of iron oxide from the bulk (macroscale) to nanoscale effectuates unique physical properties. Specifically, iron oxide nanoparticles become superparamagnetic when each particle consists of a single magnetic domain and thermal energy is high enough to overcome the energy barrier of magnetic flipping (typically, $<20 \mathrm{~nm}$ ). In MRI, the SPIONs generate local inhomogeneities in the magnetic field decreasing the signal. Therefore, regions in the body that contain SPIONs appear darker in MR images. This information can be used to monitor the uptake of SPIONs to predict treatment efficacy. ${ }^{2}$ Hyperthermia can be achieved with SPIONs using a rapidly alternating magnetic field. ${ }^{3,4}$ Heat is generated through the rapid rotation of the SPION itself (Brownian fluctuations, minor contribution) and the fluctuation of the magnetic moment within the SPION crystal lattice (Néel fluctuations, major contribution). Cells containing SPIONs will heat up inducing apoptosis.

The magnetic properties of the superparamagnetic core are affected by size, shape, and defect concentration, so can be tuned to achieve the desired property. The magnetism engineering work by Lee et al. shows that cobalt and nickel doped SPIONs have reduced mass magnetizations as compared to pure SPIONs. ${ }^{5}$ Interestingly, they also show the mass magnetization of manganese doped SPIONs is significantly higher. Therefore controlling dopant type and concentration in the SPION core can dramatically affect its magnetic properties. The shape and size of SPIONs also affects magnetic properties. ${ }^{6}$ As the aspect ratio of SPIONs is increased from spherical to rodlike, the coercivity dramatically increases resulting in improved MRI contrast and hyperthermic effects. Also, smaller SPIONs which have higher surface area to volume ratios provide smaller mass magnetizations than larger SPIONs owing to the increased contribution of the magnetically "dead" layer on the surface. Thus, larger SPIONs are desirable for their magnetic properties alone, but the physiological and cellular barriers imposed by the body restrict the usable sizes of SPIONs.

\subsection{SPION coating}

Biological barriers encountered by therapeutic SPIONs in the body must be bypassed to prolong the blood half-life and improve the likelihood of reaching target cells. This can be achieved through careful coating design to improve SPION stability in biological milieus. Here, we first discuss the barriers encountered by intravenously injected SPIONs including the barriers before (physiological barriers) and after (cellular barriers) they reach the cancer cell. We also discuss common materials used to bypass each of these barriers.

2.2.1. Physiological barriers-The physiological barriers include the blood, liver and spleen, kidneys, blood-brain barrier, and tumor vasculature (Figure 2). Blood is a complex, electrolyte-filled fluid composed of charged proteins such as albumin, enzymes, cells such as erythrocytes and leukocytes, and small molecules such as sugars, amino acids, hormones, and lipids. An improperly designed SPION coating can result in early release or degradation of therapeutic payload, and destabilization causing particle aggregation and potential embolism. The coating shields the SPION surface from adsorption of blood components and improves colloidal stability. Polyethylene glycol (PEG) is one of most commonly used shielding materials. Zwitterionic materials are used for the same purpose.

To avoid elimination by clearance organs (i.e., liver, kidneys, and spleen), the hydrodynamic size of the nanoparticle (combined size of the core and coating) should remain small. The reticuloendothelial system (RES) readily takes up materials greater than $100 \mathrm{~nm}$. Also, the basal lamina of the kidneys has pores of approximately $10 \mathrm{~nm}$, so materials larger than this will not be filtered out of the blood. SPIONs with hydrodynamic sizes between 10 and 100 
$\mathrm{nm}$ show greatly reduced liver and kidney uptake so are given the greatest opportunity to specifically interact with target cells. ${ }^{7}$

Circulating SPIONs must extravasate from the blood vessels to reach the target cells. Depending on the target tissue type, the junction between endothelial cells in the blood vessels can vary in size. This is especially true of the blood-brain barrier (BBB) where endothelial cells in the brain form tight junctions that severely limit the permeability of water soluble compounds. Different strategies have been developed for gaining access to brain tissue through the BBB. The vascular permeability of the BBB can be increased by administration of vasodilators such as bradykinin and histamine, or hypertonic solution of mannitol, to osmotically shrink endothelial cells. These methods are risky since the brain's natural defenses against harmful bloodborne molecules are disrupted Attaching BBB permeating molecules to SPIONs, such as PEG, CLPFFD (an amphipathic peptide), chitosan, and Chlorotoxin (CTX), ${ }^{8,9,10}$ that are actively transported into the brain through transcytosis, absorption, or receptor-mediated endocytosis, could provide a safer means of gaining access to cells in brain. In this case, the SPION coating must display these molecules to allow the interaction with receptors on endothelial cells of the BBB. Passive extravasation can only occur in discontinuous capillaries, which is the case with the enhanced permeation and retention (EPR) effect in rapidly growing tumors. Leaky blood vessels combined with a lack of lymph vessels promote the accumulation of delivered SPIONs of 30-100 $\mathrm{nm}$ at the tumor site.

2.2.2. Cellular barriers-Once SPIONs reach the tumor they must distribute throughout the tumor, be taken up by tumor cells, and localize to their intracellular site of action to perform the therapeutic function. The size and surface coating alone of the SPION have a determinant effect on the distribution and uptake. Nanoparticles with hydrodynamic sizes larger than $60 \mathrm{~nm}$ show limited penetration into tumor cells. ${ }^{11}$ Furthermore, experiments with nanoparticles and mathematical modeling reveal that cationic nanoparticles have better cell uptake but poor distribution throughout the tumor, while anionic nanoparticles distribute very well in the tumor but have poor cellular uptake. ${ }^{12}$ The better distribution of anionic nanoparticles in the tumor can be combined with high cell uptake associated with cationic nanoparticles through elegant coating design. For example, the charge-reversal strategy allows an anionic SPION to deeply penetrate a tumor and become cationic at the low $\mathrm{pH}$ of the tumor environment for high cell uptake. ${ }^{13,14}$ Once distributed throughout the tumor, there are three tiers of barriers that SPIONs must bypass for intracellular therapy: the cell membrane, the endosome/lysosome, and intracellular trafficking. Figure 3 illustrates these cellular barriers encountered by a typical therapeutic SPION.

The cell membrane consists of a negatively charged phospholipid bilayer embedded with cholesterol, glycolipids, and glycoproteins, and acts as a physical barrier. The SPION coating needs to provide a mechanism to enable the uptake of the SPION by the cell through either transcytosis across the cell membrane or endocytosis. Transcytosis across the cell membrane can be achieved with arginine-rich coatings. ${ }^{15}$ Endocytosis can be receptormediated through ligand-receptor interaction, adsorptive through non-specific interactions between the cell membrane and SPION, or fluid phase where bulk uptake of solutes occurs. Transcytosis provides direct access to the cytoplasm of the cell, but endocytosis involves uptake into an endosomal vesicle in which the $\mathrm{pH}$ is reduced to activate degradative enzymes.

The SPION coating requires a means to escape the endosome before degradative enzymes become active. Lipids have been shown to escape the endosome by fusing with and translocating through the lipid bilayer at reduced $\mathrm{pH}$. Similarly, fusogenic peptides, a class of amphiphilic molecules, escape the endosome through interaction with and disruption of 
the hydrophobic bilayer of the endosome at reduced $\mathrm{pH}$. A different mechanism of release from the endosome is through the proton sponge effect wherein a material buffers the influx of protons into the endosome, which causes osmotic swelling and eventually rupture of the endosome, releasing its contents into the cytoplasm of the cell. Materials that can be used as proton sponges, such as polyethyleneimine (PEI), generally contain tertiary amines which have a $\mathrm{pK}_{\mathrm{a}}$ around 5. These materials can be integrated into the SPION coating to endow the endosomal escape property. Finally, once the SPION is free in the cytoplasm of the cell, it must be delivered to the subcellular organelle to elicit its function. DNA must localize into the nucleus to be transcribed into mRNA. siRNA functions outside the nucleus on mRNA so nuclear entry is not needed. Small-molecule drugs have various intracellular therapeutic targets, and should be transported to those sites. Similarly, therapeutic proteins have different sites of action, so appropriate intracellular trafficking needs to be employed.

\subsection{Tumor targeting}

Tumor targeting refers to the selective accumulation and uptake of therapy in the tumor. Targeting of tumor cells can be achieved passively through the EPR effect or actively through use of a targeting agent. While passive tumor targeting significantly enhances uptake of nanosized therapies, active targeting provides both better uptake and distribution in the tumor. Generally, a targeting SPION is prepared by attaching a ligand that recognizes specific receptors highly expressed only on cancer cells. ${ }^{16}$ The increased metabolic function of cancer cells can also be exploited for targeting through attachment of molecules such as glucose or folic acid. ${ }^{17,18}$ Here, the specific interactions between the targeting ligand and cell surface receptors must outweigh the non-specific interactions between the SPION and cells. Thus, the SPION core should be sufficiently coated to prevent non-specific cell binding of the SPION. Further, the coating must be near charge-neutral to prevent nonspecific electrostatic interactions between cells and the SPION. PEG is commonly used as the coating material of SPIONs since it improves circulation time in blood and displays targeting agents on its termini for cell recognition.

Studies have shown that targeting agents improve the uptake of nanoparticles by tumors in both xenograft and transgenic mouse models. Recent findings indicate that some targeting agents do not actually increase particle uptake into the tumor, but improve particle distribution in the tumor and within the tumor cells. ${ }^{19}$ Both targeted anionic ${ }^{20}$ and cationic ${ }^{21}$ nanoparticles have shown this effect indicating that the improved tumor distribution and cell uptake is not associated with the surface charge. This improved particle distribution provides significant advantages for imaging and drug delivery: tumor boundaries can be better delineated and the delivered therapy can affect a larger proportion of tumor cells.

Depending on the targeting ligand used, SPIONs can be targeted to specific cells of the tumor microenvironment. RGD is a commonly used tumor targeting peptide that recognizes $\alpha_{\mathrm{v}} \beta_{3}$ integrins overexpressed on cancer cell and associated endothelial cell surfaces. CTX is a peptide that recognizes MMP-2 overexpressed on the surface of cancer cells. PEG coated SPIONs activated with CTX (NP-CTX) or RGD (NP-RGD) both provide significant contrast in MRI, but NP-RGD localizes to neovasculature whereas NP-CTX distributes throughout the tumor (Figure 4). ${ }^{22}$ Thus, the choice of targeting ligand for delivery of therapeutic drugs or biomolecules needs to match the target of the delivered therapy.

\section{Therapeutic SPIONs in cancer treatment}

In this section we present several examples of therapeutic SPIONs and discuss how the design considerations shown above are implemented in their structures for gene therapy, chemotherapy, protein therapy, and magnetic hyperthermia-based therapy. Most SPION 
formulations are still in pre-clinical stages of development, majority of them were tested in rodents with xenograft tumors.

\subsection{Gene therapy}

Gene therapy, to its simplest approximation, can mediate cancer by treating the root of the disease, namely, DNA damage that leads to aberrant expression of proteins involved in key regulatory pathways. Normal cell behavior can be restored through DNA delivery to replace the damaged gene. Similarly, over-expression of oncogenes that promote carcinogenesis can be down-regulated through RNA interference (RNAi) by delivery of small interfering RNAs (siRNAs). Of the many materials used to make non-viral vectors (e.g. liposomes, cationic polymers, dendrimers), solid core nanoparticles such as SPIONs provide the advantage of small, controllable size. The SPION core serves as a template for organization of the polymeric coating that will bind or encapsulate the nucleic acid.

Nanoparticles designed for gene therapy are generally cationic since the positively charged nanoparticles interact with negatively charged nucleic acids to form stable complexes. This is especially true for delivery of plasmid DNA which is relatively large $(\sim 100 \mathrm{~nm})$ even when compacted $(\sim 10 \mathrm{~nm})$. On the other hand, siRNA, which is small and rigid, is less restrictive on polymer coating. Once the nucleic acid bound SPION is internalized, DNA delivery requires access to the nucleus for successful transfection whereas siRNA only needs access to the cytoplasm to inhibit translation of mRNA. Cationic materials generally show good nuclear localization and peptides that promote localization to the nucleus are also used. SPIONs for gene therapy are still in pre-clinical stages of development.

SPIONs coated with a copolymer of PEI, PEG, and chitosan (namely, NP-CP-PEI) are able to stably bind plasmid DNA, protect it from external molecules such as nucleases, and deliver it to the nucleus for transfection. ${ }^{23}$ The combination of PEI and chitosan improves the low transfection efficiency associated with chitosan and suppresses the toxicity associated with PEI, and PEG passivates the SPION surface for improved stability and further diminishes PEI toxicity. Furthermore, this copolymer coating allows the SPIONs to retain their magnetic properties even when DNA is bound. These SPIONs are able to deliver green fluorescent protein encoding plasmid DNA to brain tumor cells in vivo for transfection through the EPR effect.

Targeted gene therapy can dramatically improve transfection efficiencies and treat a higher proportion of cells. Attachment of the targeting ligand, CTX, to NP-CP-PEI increases the transfection efficiency 3 -fold. ${ }^{21,24}$ The targeting SPION provides improved therapeutic efficacy even though both non-targeting and targeting SPIONs show similar contrast enhancement in MRI (Figure 5). Similarly, attachment of the peptide EPPT which recognizes the tumor-specific antigen uMUC-1 to SPIONs improves siRNA delivery to breast cancer cells. ${ }^{25}$ Cross-linked dextran is used as the particle coating to provide sufficient functional groups for covalent attachment of siRNAs and targeting agents. This SPION is able to deliver a therapeutic dose of anti-BIRC5 siRNA to human breast cancer xenograft tumors while being monitored through MRI.

The charge-reversal strategy can improve accumulation of SPIONs throughout the tumor and also provide secondary therapy. PEI is highly toxic due to its high proportion of amine groups, but becomes non-toxic when these amine groups are blocked. We attached $\mathrm{pH}$-labile blocked PEI to SPIONs along with anti-GFP siRNA and CTX. ${ }^{13}$ Under acidic pH, such as in the tumor microenvironment, the amine groups on PEI become unblocked and toxic (Figure 6a) leading to higher tumor cell kill and greater gene knockdown due to greater uptake of the SPION (Figure 6b). 


\subsection{Chemotherapy}

Chemotherapy aims to inhibit cancer cell growth through delivery of small molecule inhibitors of cell function. Many chemotherapeutic drugs inhibit DNA replication or repair so need access to the nucleus for function. Others inhibit mitochondrial activity, cell motility, and the components of the cell division pathway. Intracellular trafficking of these delivered drugs to their site of action significantly improves therapeutic potency.

Failure of chemotherapy is generally caused by limited dose that can be applied due to offtarget toxicity, and acquired drug resistance in cancer cells. The use of nanoparticles for delivery of chemotherapies can provide significant advantages over drug alone. The multifunctionality of nanoparticles offers better biodistribution of drug allowing for administration of higher doses. ${ }^{26}$ Furthermore, drug attached to nanoparticles can overcome the multi-drug resistance (MDR) by many cancers in which drug efflux pumps are overexpressed on their surfaces. When attached to nanoparticles, the drug is no longer a substrate for these efflux pumps. A hydrophobic polymer coating on the SPION can be employed for loading of hydrophobic drug, or if the drug has a functional group it can be covalently linked to SPIONs.

Hydrophobic drug loading onto SPIONs can provide high drug loading efficiencies and increase drug stability under physiological conditions. For example, Jain et al. attached Pluronic F127, a PEO-PPO di-block co-polymer, on oleic acid coated SPIONs,${ }^{2}$ wherein the oleic acid/PPO layer provides a hydrophobic region for high-capacity drug loading. The passive uptake of these SPIONs in xenograft tumors through the EPR effect can be monitored using MRI.

Direct conjugation of drug to SPIONs can provide better control over drug loading and release, but loading capacity is low due to the small number of functional groups on the surface of SPIONs as compared to hydrophobic regions in hydrophobic coatings. To achieve higher drug loading a docking molecule can be used to bind large amounts of drug before attaching to SPIONs. We utilized PEI as a docking molecule for DOX attachment to SPIONs, which accommodates over 1000 DOX molecules per SPION. ${ }^{27}$ We showed that this SPION:DOX conjugate (NP-DOX) is able to overcome MDR in glioblastoma by preventing drug efflux, and provides a higher intracellular concentration of DOX for improved cell kill (Figure 7). Free DOX is much less effective in killing drug-resistant cells as compared to drug-sensitive cells with a resistance factor of around 55. On the other hand, NP-DOX is much less sensitive to drug resistance with a resistance factor of only $\sim 5$. A high resistance factor suggests high sensitivity towards drug resistance.

\subsection{Protein therapy}

The goal of protein therapy is to deliver a sufficient dose of therapeutic protein into cancer cells, which is considered one of the most direct and safe approaches for treating cancer. Protein therapy can include blocking a cell surface receptor involved in carcinogenesis, or intracellular delivery of proteins involved in cell signaling to either slow down cell growth and invasion, or induce apoptosis. However, delivery of proteins can be very challenging. Therapeutic proteins are often metabolized or cleared in vivo before reaching target cells. Nanoparticles can significantly improve protein delivery by protecting them from protease degradation and acting as a vehicle for intracellular access and trafficking. Furthermore, the therapy can be enhanced through the multivalent effect wherein protein molecules are arranged on the surface of the nanoparticle so that the effect is stronger than a single protein. For example, CTX labeled SPIONs have been shown to hinder the invasiveness of glioblastoma cells to a greater extent than free CTX owing to the multivalent effect ${ }^{9}$. 
Cell surface receptor inhibition for cancer therapy has been achieved with epidermal growth factor receptor variant III (EGFRvIII) antibody labeled SPIONs. ${ }^{28}$ These SPIONs are able to specifically inhibit the function of EGFRvIII to induce apoptosis in glioma cells in vitro, which translated into improved time of survival in vivo after convection enhanced delivery of SPIONs into the brain.

SPIONs can also deliver therapeutic doses of protein intracellularly; however, these SPIONs are still in early stages of development and many have not been tested in cells. For example, cytochrome $\mathrm{c}$ induces apoptosis once it is internalized, but is poorly taken up by cells. Cytochrome c protein loaded into nanoparticles increases its cytoplasmic concentration and induces apoptosis in breast cancer cells. ${ }^{29}$ SPIONs have also been used to adsorb cytokines such as interferon gamma (IFN $\gamma$ ) for potential cancer immunotherapy. ${ }^{30}$ The surface of these SPIONS was engineered to be highly anionic for adsorption of cationic IFN $\gamma$. This strategy enables efficient protein loading and release of active cytokine.

\subsection{Magnetic hyperthermia}

The magnetic properties of SPIONs can be exploited to induce a therapeutic response through hyperthermia. In an alternating magnetic field, SPIONs generate heat primarily through Néel fluctuations which can result in very high local temperatures. Jordan et al. exploited this property for cancer therapy. ${ }^{3}$ They showed that aminosilane coated SPIONs are able to increase survival in rats with orthotopic brain tumors through intratumoral injection of SPIONs and subsequent thermotherapy. The aminosilane coating likely promotes electrostatic interaction between the SPION and cell membrane to prevent leakage out of the tumor. However, the high concentration of SPIONs needed in the tumor tissue currently limits the broad application of this technology to solid tumors that can be accessed through injection. The tolerability of this therapy in glioblastoma patients has been shown in a Phase I clinical trial suggesting it could work well in these solid tumors. ${ }^{31}$

Alternatively, magnetic hyperthermia can be used to selectively release drug. Thomas et al. loaded mesoporous silica nanoparticles with DOX and SPIONs and capped the pores of the silica with a heat labile molecule (Figure 8a). ${ }^{32}$ At room temperature, there is no drug release since the pores of the mesoporous silica remain capped, but at increased temperatures obtained through magnetic hyperthermia, de-capping occurs releasing most of the drug. This controlled release provides 7-times higher cell kill than without hyperthermic release in vitro (Figure $8 \mathrm{~b}$ ). In a clinical setting, this nanodrug could provide a significant advantage over current chemotherapies since drug release could be limited to the extent of the alternating magnetic field to prevent deleterious off-target effects.

\section{Conclusion and Perspective}

SPIONs have been extensively studied as MRI contrast agents and drug carriers both in vitro and in vivo. The design parameters are critical to the success of resultant imaging and therapeutic SPIONs. The size, shape, and crystallinity of the iron oxide core all play a role in providing high contrast in MRI, and the polymer coating dictates the particle stability and the end-use of the SPION for targeted therapy. Various surface engineering strategies have been utilized to achieve improved treatment specificity and therapeutic index of the SPIONs.

The ability of SPIONs to serve as an MRI contrast agent and the prolonged accumulation of SPIONs in tumors (e.g., weeks) allows physicians to non-invasively and continuously assess tumor-targeted therapeutic delivery, distribution, uptake, and predict response in patients immediately after treatment so that dosing can be personalized for more effective treatment. With the potential of detecting single cells labeled with SPIONs under high magnetic strength, very specific information on delivery can be obtained. 
Currently, several SPION formulations such as Ferridex I.V., Ferumoxytol, and Combidex are approved for use in the clinic, but are non-targeted and provide only diagnostic information. These formulations do, however, act as a stepping stone onto which other SPION nanomedicine formulations can be developed since the safety profile of the base SPION core has been established. SPIONs combined with clinically used drugs are expected to be the forerunner for the translation of laboratory results to clinic.

Highly reproducible synthesis of multi-functional SPIONs and integration of multiple design components remain challenging. For eventual translation to the clinic, the focus must be placed on the homogeneity of developed SPIONs so that desired properties such as size and shape of SPION, numbers of functional groups present, and drug loading and release, can be well controlled. To accommodate multiple types of biomolecules in one nanoparticle design, one component that can serve multiple functions is preferable to simplify implementation and would have less hurdles to get FDA approval. Moreover, in vivo nanoparticle targeting to achieve therapeutic efficacy should be demonstrated in large animals as targeted experiments in rodents may not be readily reproduced in larger animals/human. Future emphasis should be placed on surface engineering of SPIONs to reduce nonspecific SPION uptake by non-targeted organs and increase their uptake by target tumors. Finally, a rigorous characterization of SPIONs with focus on addressing the potential regulatory issues would be needed to achieve their speedy translation to the clinic.

\section{Acknowledgments}

We acknowledge the support of the NIH Grants R01EB006043, R01CA134213 and T32CA138312.

\section{References}

1. Sun C, Du K, Fang C, Bhattarai N, Veiseh O, Kievit F, Stephen Z, Lee D, Ellenbogen RG, Ratner B, Zhang M. PEG-mediated synthesis of highly dispersive multifunctional superparamagnetic nanoparticles: their physicochemical properties and function in vivo. ACS Nano. 2010; 4:2402-10. [PubMed: 20232826]

2. Jain TK, Foy SP, Erokwu B, Dimitrijevic S, Flask CA, Labhasetwar V. Magnetic resonance imaging of multifunctional pluronic stabilized iron-oxide nanoparticles in tumor-bearing mice. Biomaterials. 2009; 30:6748-56. [PubMed: 19765817]

3. Jordan A, Scholz R, Maier-Hauff K, van Landeghem FKH, Waldoefner N, Teichgraeber U, Pinkernelle J, Bruhn H, Neumann F, Thiesen B, von Deimling A, Felix R. The effect of thermotherapy using magnetic nanoparticles on rat malignant glioma. J Neuro-Oncol. 2006; 78:714.

4. Day ES, Morton JG, West JL. Nanoparticles for thermal cancer therapy. J Biomech Eng. 2009; 131:074001. [PubMed: 19640133]

5. Lee JH, Huh YM, Jun YW, Seo JW, Jang JT, Song HT, Kim S, Cho EJ, Yoon HG, Suh JS, Cheon J. Artificially engineered magnetic nanoparticles for ultra-sensitive molecular imaging. Nat Med. 2007; 13:95-9. [PubMed: 17187073]

6. Lu AH, Salabas EL, Schuth F. Magnetic nanoparticles: synthesis, protection, functionalization, and application. Angew Chem Int Ed Engl. 2007; 46:1222-44. [PubMed: 17278160]

7. Longmire M, Choyke PL, Kobayashi H. Clearance Properties of Nano-Sized Particles and Molecules as Imaging Agents: Considerations and Caveats. Nanomedicine. 2008; 3:703-717. [PubMed: 18817471]

8. Guerrero S, Araya E, Fiedler JL, Arias JI, Adura C, Albericio F, Giralt E, Arias JL, Fernandez MS, Kogan MJ. Improving the brain delivery of gold nanoparticles by conjugation with an amphipathic peptide. Nanomedicine (Lond). 2010; 5:897-913. [PubMed: 20735225]

9. Veiseh O, Sun C, Fang C, Bhattarai N, Gunn J, Kievit F, Du K, Pullar B, Lee D, Ellenbogen RG, Olson J, Zhang M. Specific targeting of brain tumors with an optical/magnetic resonance imaging nanoprobe across the blood-brain barrier. Cancer Res. 2009; 69:6200-7. [PubMed: 19638572] 
10. Hombach J, Bernkop-Schnurch A. Chitosan solutions and particles: evaluation of their permeation enhancing potential on MDCK cells used as blood brain barrier model. Int J Pharm. 2009; 376:104-9. [PubMed: 19409469]

11. Popovic Z, Liu W, Chauhan VP, Lee J, Wong C, Greytak AB, Insin N, Nocera DG, Fukumura D, Jain RK, Bawendi MG. A nanoparticle size series for in vivo fluorescence imaging. Angew Chem Int Ed Engl. 2010; 49:8649-52. [PubMed: 20886481]

12. Kim B, Han G, Toley BJ, Kim CK, Rotello VM, Forbes NS. Tuning payload delivery in tumour cylindroids using gold nanoparticles. Nat Nanotechnol. 2010; 5:465-72. [PubMed: 20383126]

13. Mok H, Veiseh O, Fang C, Kievit FM, Wang FY, Park JO, Zhang M. pH-Sensitive siRNA Nanovector for Targeted Gene Silencing and Cytotoxic Effect in Cancer Cells. Mol Pharm. 2010

14. Mok H, Park JW, Park TG. Enhanced intracellular delivery of quantum dot and adenovirus nanoparticles triggered by acidic $\mathrm{pH}$ via surface charge reversal. Bioconjug Chem. 2008; 19:797801. [PubMed: 18363345]

15. Futaki S, Suzuki T, Ohashi W, Yagami T, Tanaka S, Ueda K, Sugiura Y. Arginine-rich peptides. An abundant source of membrane-permeable peptides having potential as carriers for intracellular protein delivery. J Biol Chem. 2001; 276:5836-40. [PubMed: 11084031]

16. Sun C, Veiseh O, Gunn J, Fang C, Hansen S, Lee D, Sze R, Ellenbogen RG, Olson J, Zhang M. In vivo MRI detection of gliomas by chlorotoxin-conjugated superparamagnetic nanoprobes. Small. 2008; 4:372-9. [PubMed: 18232053]

17. Sun C, Sze R, Zhang M. Folic acid-PEG conjugated superparamagnetic nanoparticles for targeted cellular uptake and detection by MRI. J Biomed Mater Res A. 2006; 78:550-7. [PubMed: 16736484]

18. Meier R, Henning TD, Boddington S, Tavri S, Arora S, Piontek G, Rudelius M, Corot C, DaldrupLink HE. Breast cancers: MR imaging of folate-receptor expression with the folate-specific nanoparticle P1133. Radiology. 2010; 255:527-35. [PubMed: 20413763]

19. Bartlett DW, Su H, Hildebrandt IJ, Weber WA, Davis ME. Impact of tumor-specific targeting on the biodistribution and efficacy of siRNA nanoparticles measured by multimodality in vivo imaging. Proc Natl Acad Sci US A. 2007; 104:15549-54.

20. Choi CH, Alabi CA, Webster P, Davis ME. Mechanism of active targeting in solid tumors with transferrin-containing gold nanoparticles. Proc Natl Acad Sci US A. 2010; 107:1235-40.

21. Kievit FM, Veiseh O, Fang C, Bhattarai N, Lee D, Ellenbogen RG, Zhang M. Chlorotoxin labeled magnetic nanovectors for targeted gene delivery to glioma. ACS Nano. 2010; 4:4587-94. [PubMed: 20731441]

22. Fang C, Veiseh O, Kievit F, Bhattarai N, Wang F, Stephen Z, Li C, Lee D, Ellenbogen RG, Zhang M. Functionalization of iron oxide magnetic nanoparticles with targeting ligands: their physicochemical properties and in vivo behavior. Nanomedicine (Lond). 2010; 5:1357-69. [PubMed: 21128719]

23. Kievit FM, Veiseh O, Bhattarai N, Fang C, Gunn JW, Lee D, Ellenbogen RG, Olson JM, Zhang M. PEI-PEG-Chitosan Copolymer Coated Iron Oxide Nanoparticles for Safe Gene Delivery: synthesis, complexation, and transfection. Adv Funct Mater. 2009; 19:2244-2251. [PubMed: 20160995]

24. Veiseh O, Kievit FM, Fang C, Mu N, Jana S, Leung MC, Mok H, Ellenbogen RG, Park JO, Zhang M. Chlorotoxin bound magnetic nanovector tailored for cancer cell targeting, imaging, and siRNA delivery. Biomaterials. 2010; 31:8032-42. [PubMed: 20673683]

25. Kumar M, Yigit M, Dai G, Moore A, Medarova Z. Image-guided breast tumor therapy using a small interfering RNA nanodrug. Cancer Res. 2010; 70:7553-61. [PubMed: 20702603]

26. Hua MY, Yang HW, Chuang CK, Tsai RY, Chen WJ, Chuang KL, Chang YH, Chuang HC, Pang ST. Magnetic-nanoparticle-modified paclitaxel for targeted therapy for prostate cancer. Biomaterials. 2010; 31:7355-63. [PubMed: 20609471]

27. Kievit FM, Wang FY, Fang C, Mok H, Wang K, Silber JR, Ellenbogen RG, Zhang M. Doxorubicin loaded iron oxide nanoparticles overcome multidrug resistance in cancer in vitro. $\mathrm{J}$ Control Release. 10.1016/j.jconrel.2011.01.024

28. Hadjipanayis CG, Machaidze R, Kaluzova M, Wang L, Schuette AJ, Chen H, Wu X, Mao H. EGFRvIII antibody-conjugated iron oxide nanoparticles for magnetic resonance imaging-guided 
convection-enhanced delivery and targeted therapy of glioblastoma. Cancer Res. 2010; 70:630312. [PubMed: 20647323]

29. Santra S, Kaittanis C, Perez JM. Cytochrome C encapsulating theranostic nanoparticles: a novel bifunctional system for targeted delivery of therapeutic membrane-impermeable proteins to tumors and imaging of cancer therapy. Mol Pharm. 2010; 7:1209-22. [PubMed: 20536259]

30. Mejias R, Costo R, Roca AG, Arias CF, Veintemillas-Verdaguer S, Gonzalez-Carreno T, del Puerto Morales M, Serna CJ, Manes S, Barber DF. Cytokine adsorption/release on uniform magnetic nanoparticles for localized drug delivery. J Control Release. 2008; 130:168-74. [PubMed: 18588929]

31. Maier-Hauff K, Rothe R, Scholz R, Gneveckow U, Wust P, Thiesen B, Feussner A, von Deimling A, Waldoefner N, Felix R, Jordan A. Intracranial thermotherapy using magnetic nanoparticles combined with external beam radiotherapy: results of a feasibility study on patients with glioblastoma multiforme. J Neurooncol. 2007; 81:53-60. [PubMed: 16773216]

32. Thomas CR, Ferris DP, Lee JH, Choi E, Cho MH, Kim ES, Stoddart JF, Shin JS, Cheon J, Zink JI. Noninvasive remote-controlled release of drug molecules in vitro using magnetic actuation of mechanized nanoparticles. J Am Chem Soc. 2010; 132:10623-5. [PubMed: 20681678]

\section{Biographies}

Forrest Kievit is a Ph.D. student in Department of Materials Science \& Engineering at University of Washington. His research focuses on development of nanomaterials for cancer therapy.

Miqin Zhang is a professor in Department of Materials Science \& Engineering at University of Washington. She received her Ph.D. in 1999 from University of California at Berkeley. Her research focuses on nanomaterials for cancer diagnosis and treatment, biodegradable scaffolds for tissue engineering, and biosensors for detection of chemical and biological agents. 

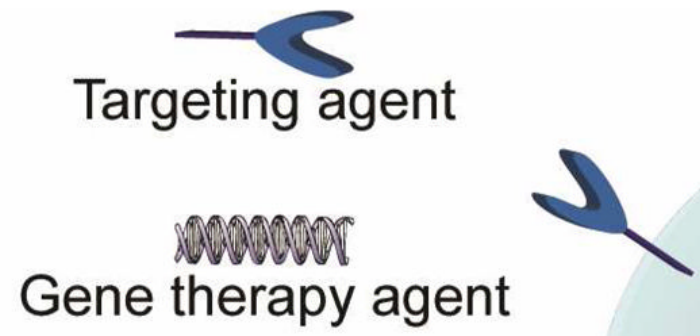

Therapeutic protein

Chemotherapy drug

Biocompatible coating

\section{菠 \\ Magnetic hyperthermia}

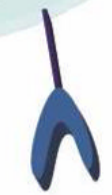

Figure 1.

Architecture of a nanotherapeutic SPION. 


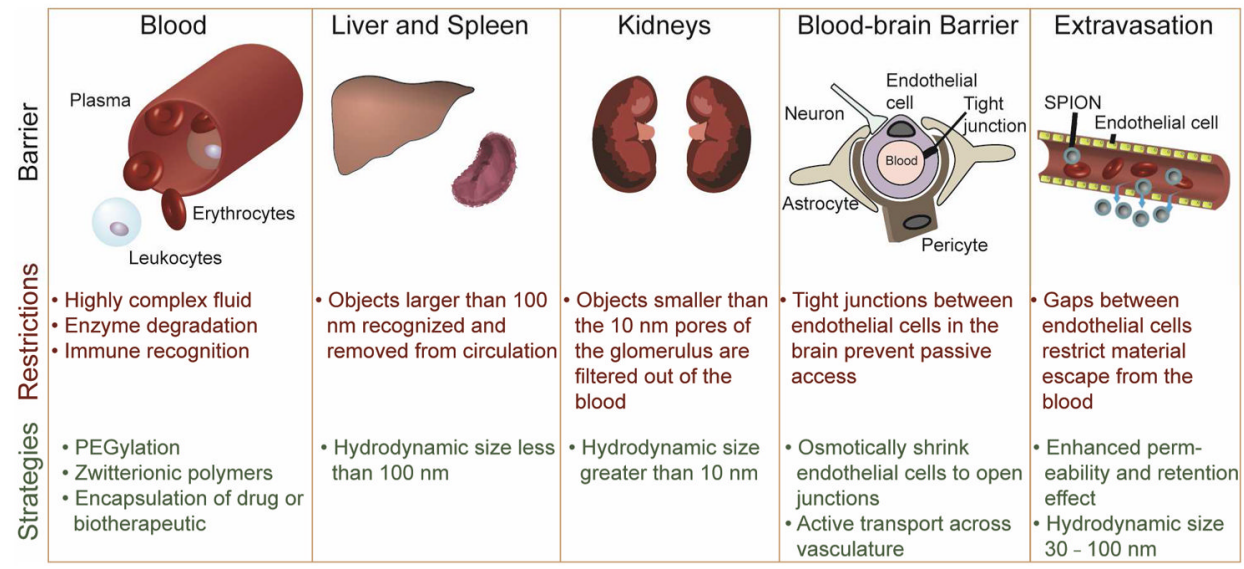

Figure 2.

Physiological barriers encountered by a typical therapeutic nanoparticle. 


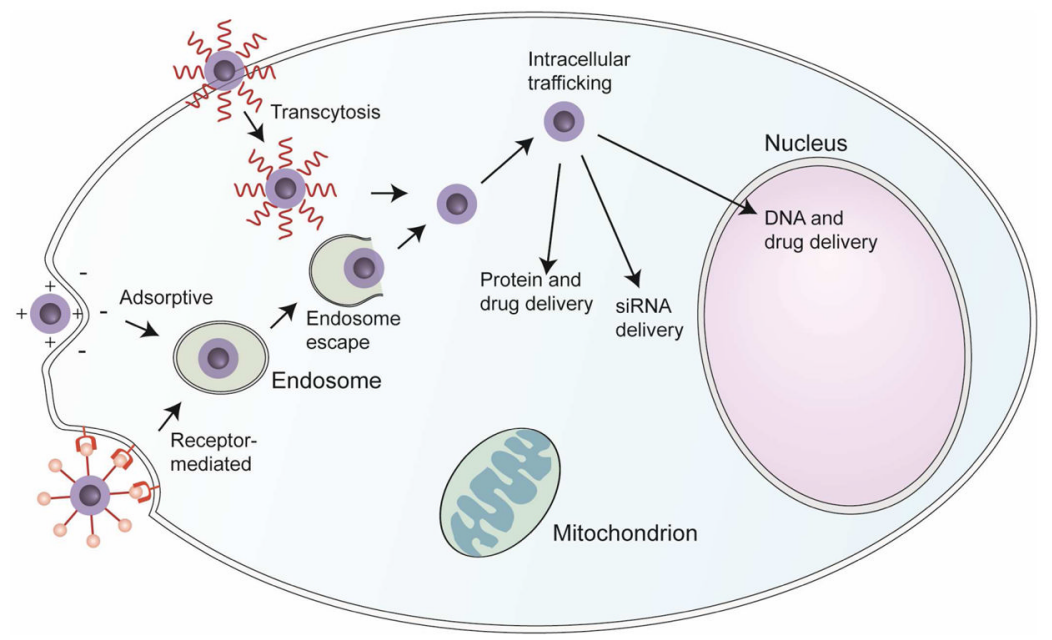

Figure 3.

Cellular barriers encountered by a typical therapeutic nanoparticle. 
a

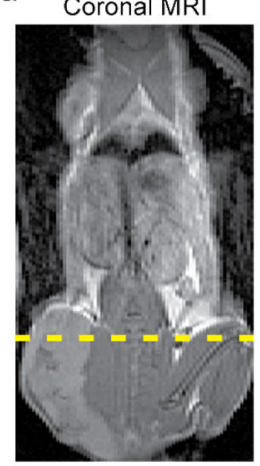

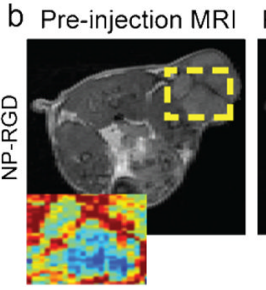
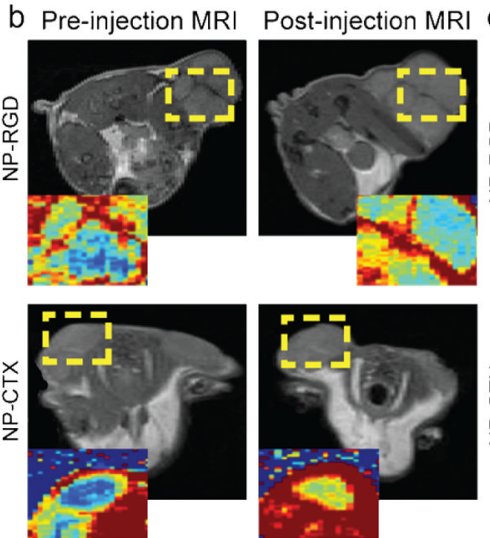

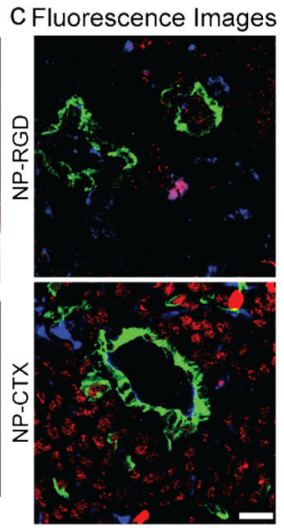

Figure 4.

Targeting SPIONs in the tumor microenvironment. a) Coronal MR image of a mouse bearing xenograft tumors. b) MR images of NP-CTX and NP-RGD treated mice. c) Histological analysis of tumors showing the selective localization of NP-RGD with neovasculature and NP-CTX throughout the tumor. Color scheme for c: green, anti-CD31 (mature endothelial cell marker); blue, anti-CD61 (neovasculature marker); red, nanoparticles. Panels $b$ and $c$ adapted with permission from ref. ${ }^{22}$ Copyright 2010 Future Medicine. 


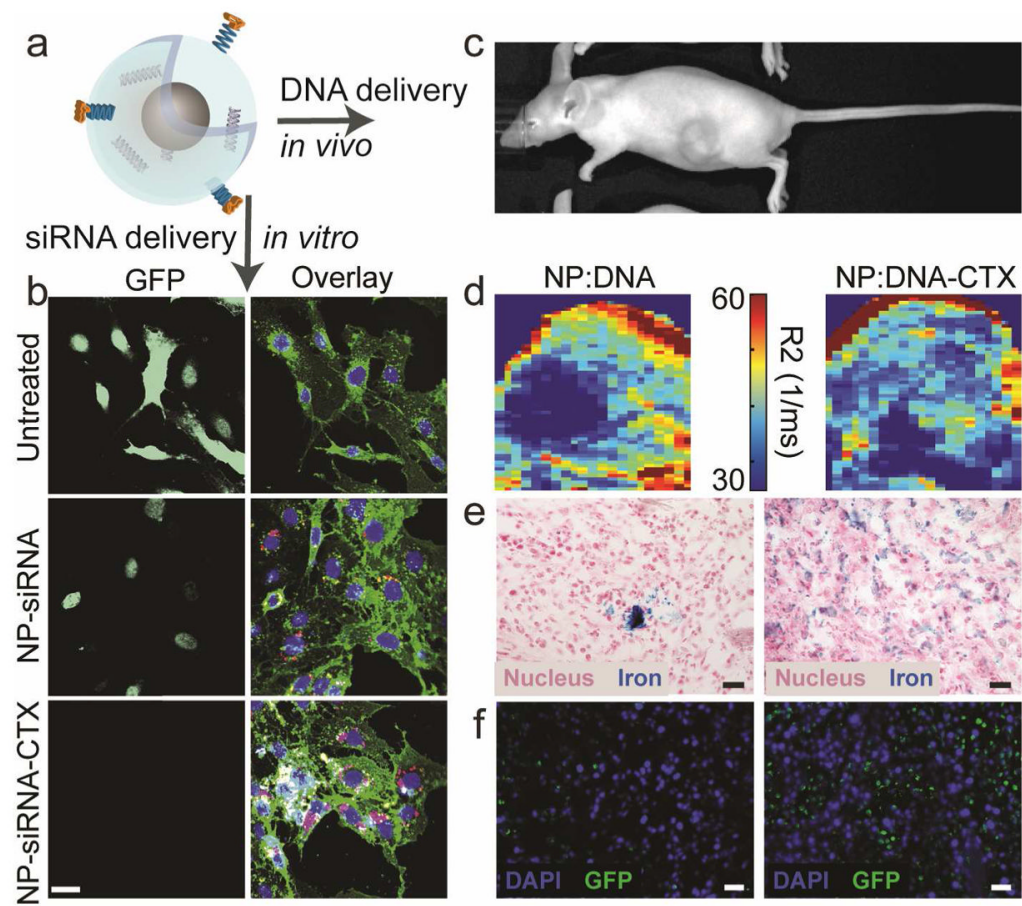

Figure 5.

CTX-targeted gene delivery using SPIONs. a) SPION activated with CTX for siRNA delivery in vitro and DNA delivery in vivo. b) Fluorescence images of GFP expressing cells treated with nanoparticles. Color scheme: blue, nucleus; green, membrane; red, siRNA; light green, GFP. c) Photograph of a typical xenograft mouse used for in vivo DNA delivery experiments. d) R2 map of xenograft brain tumors in mice treated with control (NP:DNA) and targeting (NP:DNA-CTX) SPIONs. e) Prussian blue stained xenograft brain tumors and f) fluorescence images of xenograft brain tumors, both showing the wider distribution of NP:DNA-CTX corresponds to higher expression of the delivered GFP gene. Scale bars correspond to $20 \mu \mathrm{m}$. Adapted with permission from refs. ${ }^{21,24}$ Copyright 2010 ACS and 2010 Elsevier. 


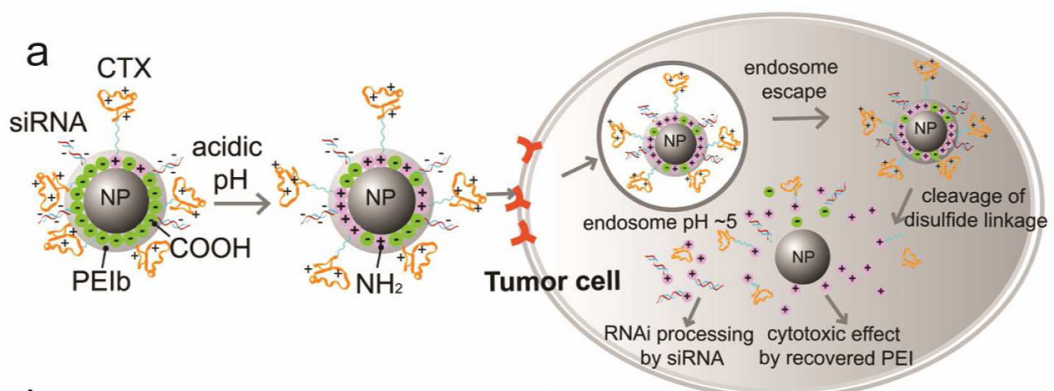

b

C

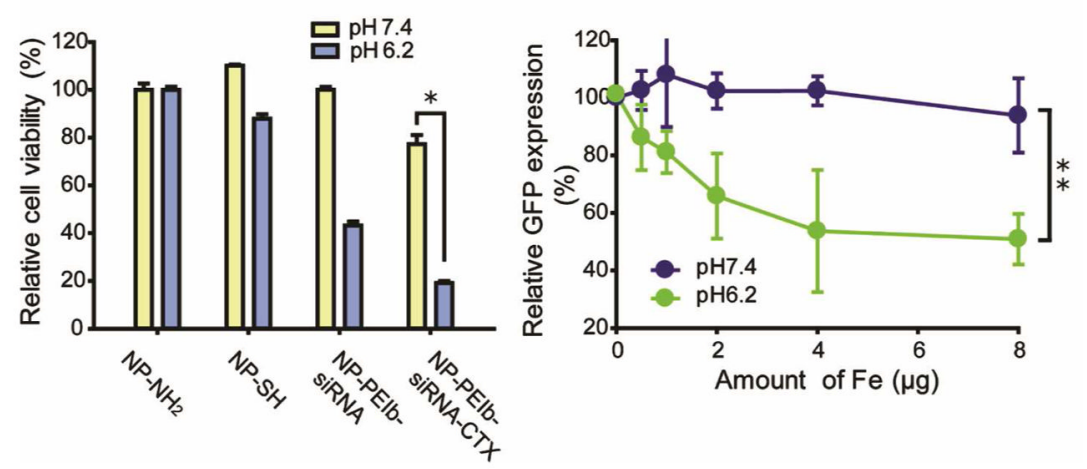

Figure 6.

Charge-reversal strategy for dual RNAi and cell-killing therapy. a) Proposed mechanism of charge-reversal strategy. b) Cell viability after treatment with various SPION formulations at neutral $\mathrm{pH}$ and acidic $\mathrm{pH}$. c) GFP expression in cells treated with the CTX-targeted SPION under the two $\mathrm{pH}$ conditions. Adapted with permission from ref. ${ }^{13}$ Copyright 2010 ACS. 

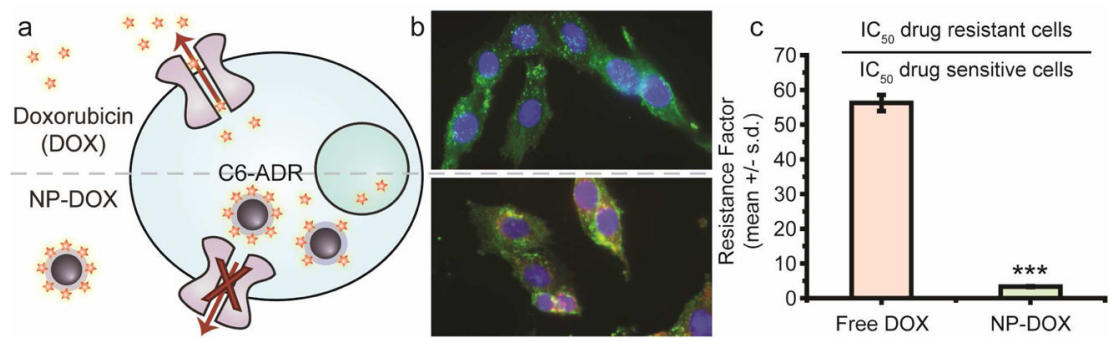

Figure 7.

DOX-loaded SPIONs for overcoming MDR. a) Cartoon showing that drug resistant glioma cells (C6-ADR) overexpressing efflux pumps are able to pump out free DOX whereas DOX attached to SPIONs remains inside the cell. DOX is then released from the SPION to intercalate DNA in the nucleus, killing the cell. b) Fluorescence images of C6-ADR cells treated with free DOX (top) and NP-DOX (bottom). c) Resistance factor for free DOX and NP-DOX. Reproduced with permission from ref. ${ }^{27}$ Copyright 2011 Elsevier. 


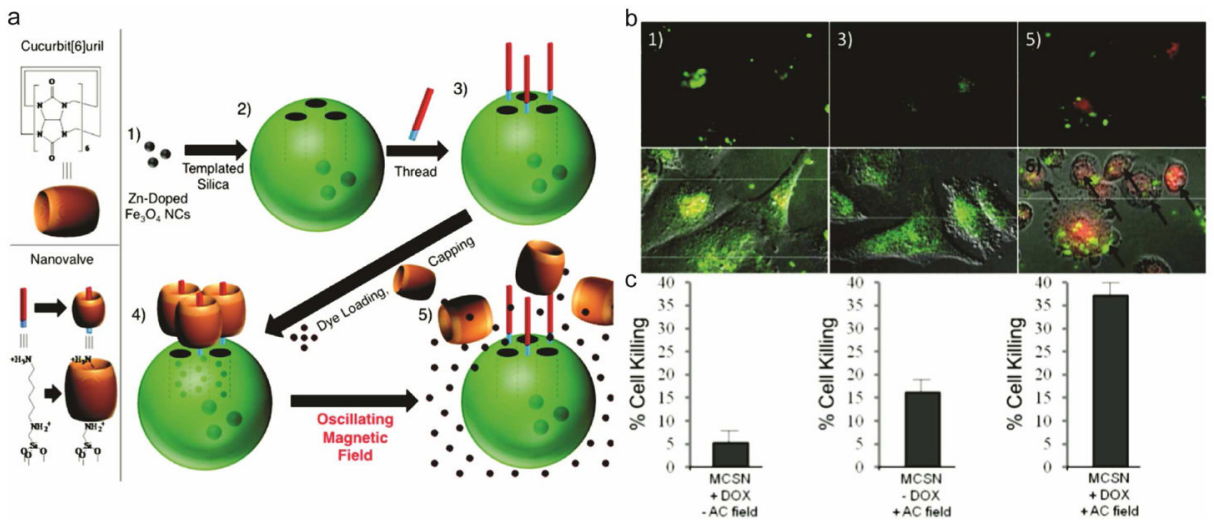

Figure 8.

Magnetically-activated release system (MARS) for breast cancer cell treatment. (a) MARS schematic. (b) Fluorescent images (top row) and fluorescent images with differential interference contrast (bottom row) of cancer cells treated with DOX-loaded MARS without AC field $(1,2)$, empty MARS with AC field $(3,4)$, and DOX-loaded MARS with AC field $(5,6)$. Color scheme: green, fluorescently-labeled MARS; red, doxorubicin (DOX). The black arrows in image 6 indicate the location of apoptotic cells. (c) Cell kill associated with each treatment. Adapted with permission from ref. ${ }^{32}$ Copyright 2010 ACS. 